\title{
Prácticas Territoriales en el IUNMa: Una propuesta integral para hacer (y ser) la Universidad que deseamos
} Territorial Internships at the IUNMa: A comprehensive
proposal to make (and be) the University we want

Anabel Pascual*

https://orcid.org/0000-0002-2081-8139

Paula Abdala**

https://orcid.org/0000-0001-8437-0751

practicasterritorialesiunma@gmail.com

Instituto Universitario Nacional de Derechos Humanos Madres de Plaza de Mayo I Argentina

\section{RESUMEN}

Planificar y reflexionar sobre la integración de las funciones desde la currícula anual y obligatoria de Prácticas Territoriales, de la licenciatura en Comunicación del Instituto Universitario Nacional de Derechos Humanos Madres de Plaza de Mayo (IUNMa), nos ubica en el ejercicio de la sistematización de nuestras prácticas docentes y pedagógicas, poniendo el foco en la importancia de los y las estudiantes como sujetos activos del proceso de enseñanza y aprendizaje pero sobre todo, en la necesidad de una universidad abierta que rescate su historia, que revalorice y construya desde su propia identidad, desde sus contextos y actores sociales del territorio que la habitan, para conformar una universidad por y para el Pueblo.

\section{ABSTRACT}

Planning and reflecting on the integration of functions from the annual and mandatory curriculum of Territorial Practices, of the degree in Communication of the National University Institute of Human Rights Mothers of the Plaza de Mayo (IUNMa), places us in the exercise of the systematization of our teaching and pedagogical practices, focusing on the importance of students as active subjects in the teaching and learning process but above all, on the need for an open university that rescues its history, revalues and builds from its own identity, from their contexts and social actors of the territory that inhabit it, to form a university by and for the People.

PALABRAS CLAVE Prácticas territoriales, Integración, identidad, Universidad
ABSTRACT

Territorial practices, Integration, identity, University 
EL INSTITUTO UNIVERSITARIO DE LES HIJES Y NIETES. LA LEY DE CREACIÓN Y SU FUERZA DE ACCIÓN.

El Instituto Universitario Nacional de Derechos Humanos "Madres de Plaza de Mayo" creado bajo la ley 26.995 en el 2014 tiene como principios rectores la formación en el área disciplinar de los derechos humanos, con orientaciones que permitan lograr un perfil de egresados/as altamente formados en la materia, para lo cual circunscribe su oferta académica de formación profesional, de investigación, de producción y de extensión, al área disciplinaria de las Humanidades y Ciencias Sociales con orientación en Derechos Humanos.

En su estatuto, en el artículo 5, se afirma que entiende como misión irrenunciable de las instituciones universitarias nacionales el contribuir al desarrollo nacional, al mejoramiento de las condiciones de vida del pueblo, a la democratización de la vida colectiva, a la resolución de los problemas concretos que aquejan a la comunidad, a la creación y difusión de un conocimiento socialmente útil en el marco del diálogo entre el saber académico y las culturas populares.

Estas normativas marco permitieron la creación de programas y proyectos curriculares que prioricen las actividades de enseñanza desde las pedagogías críticas, promoviendo así el trabajo mancomunado entre educadores y educandos desde una perspectiva situada culturalmente en lo local, regional y nacional.

En este sentido, sostener que "existe un derecho a la Universidad" (Rinesi, E. 2015, pág 96) significa postular que ésta institución y sus actores intervinientes tiene la obligación de reconocer en sus estudiantes a los sujetos de ese derecho, pero "no puede ser pensado apenas como un derecho individual ni como la suma de derechos de no importan cuantos cientos de miles de individuos (estudiantes universitarios o de ciudadanos con la posibilidad de convertirse en estudiantes universitarios), sino que debe ser pensado, también, como 
de el autor es comprendido como el conjunto de organizaciones territoriales con la que se vincula la Universidad desde mediaciones colectivas a través de las cuales se define el sentido mismo de su vida en común (la negrita es propia).

De ésta manera, la Universidad como bien colectivo de la nación y como patrimonio común del pueblo que prioriza la democracia participativa y la ciudadanía comunicativa (Mata, 2006; Teixeira, 2019), la "libertad para" por sobre "la libertad de", que permiten comprometer los mecanismos necesarios hacia una racionalidad científica que Teixeira entiende como metodología de resistencia la cual construye los caminos para la constitución de la ciudadanía comunicativa, en el seno de los movimientos de resistencia social.

Desde estos postulados teóricos entendemos que la valoración y la ideología son constitutivas del conocimiento social y "están ancladas en las condiciones sociales y materiales variadas en que se encuentran los sujetos, por las cuales las condiciones sociales se hacen diferenciables entre sí" (Follari, R. 2000 pág. 118) resultando prioritarias en la construcción del conocimiento con acuerdo social que postula la materia Prácticas Territoriales I, II y III, de currícula anual y obligatoria.

Desde el 2018 (año de ingreso de las docentes titulares que presentan ésta sistematización) se pretende contribuir a la formación de redes comunitarias que permitan la ampliación de derechos y a la circulación del conocimiento, que se refleja en el relevamiento de problemas sociales detectados por estudiantes, organizaciones sociales y docentes $y$, que se sustentan en la elaboración de diagnósticos participativos como así también en la formulación de posibles soluciones que se presentan como conocimiento útil que enriquece la materia y permite además trazar posibles líneas de investigación, e incluso aportar a la agenda del Ministerio de Justicia y Derechos Humanos y demás organismos públicos.

\section{EXTENSIÓN COMO FARO RECTOR DE LA DOCENCIA E INVESTIGA- CIÓN. PRESENTACIÓN DE PRÁCTICAS TERRITORIALES.}

Prácticas Territoriales es una materia anual que posee tres niveles a desarrollarse desde primer año hasta tercero inclusive en la Licenciatura en Comunicación del Instituto Universitario Nacional de Derechos Humanos Madres de Plaza de Mayo. La misma funciona desde la modalidad taller y nos invita a la experiencia de habitar (aprendiendo) un lugar, nos proporciona la posibilidad de permanecer en él, de entrar en el continuo diálogo entre los sujetos de la educación y de la acción social, con el objetivo de problematizar desde la comunicaciòn para generar procesos de desarrollo.

Desde su propuesta curricular se consolidan metodologías participativas que integran la universidad y con el pueblo, con la clara in- 
tención de generar procesos de transformación que, desde la acción colectiva y el diálogo de saberes, tiendan a mejorar la calidad de vida.

El diálogo de saberes se convierte en una opción posible, viable y necesaria para producir conocimiento desde dicho proyecto curricular, porque en ella se concentran la diversidad de espacios pero también la diversidad de perspectivas y de miradas sobre el mundo, que promueven la problematización de la realidad.

\section{El diálogo de saberes se convierte en una opción posible, via- ble y necesaria para producir conocimiento desde dicho pro- yecto curricular, porque en ella se concentran la diversidad de espacios pero también la diversidad de perspectivas y de miradas sobre el mundo, que promueven la problematización de la realidad.}

En este sentido se torna importante el debate y diálogo reflexivo, crítico, entre universitarios y universitarias en conjunto con el equipo docente para el desarrollo de la materia y del proyecto a continuar en el segundo y tercer año de Prácticas.

Estos talleres surgen con la impronta de la práctica extensionista entendida como la "construcción de conocimiento con acuerdo social" (Castro, J. y Oyarbide, F. 2015) que apunta a generar un proceso de producción de sentidos en el cual, el carácter sociocomunitario y socioeducativo del conocimiento que se produce no puede quedar dentro de las instituciones educativas sino que debe apuntar a generar procesos dinámicos que se encuentren en un estado permanente de diálogo con las realidades de cada territorio.

En este sentido se trabajó con los y las estudiantes al IUNMa como territorio, reconociendo por un lado la necesidad de clarificar la fuerza de la institución educativa, de su historia y actores sociales intervinientes y por otro, los años de resistencia macrista que forjaron una sólida unión entre docentes y estudiantes organizados por claustros y agrupaciones, a raíz de solidarizarse por la deuda salarial de años con les docentes y por la necesidad de resistir para que el proyecto neoliberal no vacíe la universidad de las madres. En el proceso se reconoció a la institución educativa como "un espacio dinámico, integrado por distintos actores que constantemente generan relaciones sociales con otros pares, cargando de sentido y simbolismo el territorio. Por lo que se puede inferir que el territorio forma parte de los actores, y los actores del territorio. Podría definirse como una relación simbiótica donde uno depende, coexiste y coopera con el otro". (Guillaza, V. 2020). El territorio es, por un lado, geográfico pero también simbólico, construido, disputado y puesto en tensión a través de sus actores "sin 
las relaciones sociales el territorio se desvanece y resquebraja como una hojarasca."(Stratta, F. y Barrera, M. 2009).

\section{El territorio es, por un lado, geográfico pero también simbólico, construido, disputado y puesto en tensión a través de sus acto- res "sin las relaciones sociales el territorio se desvanece y res- quebraja como una hojarasca."(Stratta, F. y Barrera, M. 2009).}

Pueyrredón 19 (dirección del edificio donde se encuentra el IUNMa) "es un espacio para reunirnos. Nosotros estamos allí desde hace 6 años más o menos, desde donde brindamos talleres, capacitaciones, asesoramiento. Tenemos un equipo interdisciplinario que atiende todos los días, al ser Once un lugar neurálgico, donde confluyen todas las colectividades. Hay compañeros y compañeras diariamente, informados por el boca a boca, enterados que hay una organización, buscando asesoramiento, contención, de todo: desde compañeros, compañeras que no saben sacar un documento, hasta mujeres víctimas de violencia familiar." El testimonio corresponde a una entrevista en profundidad a Lourdes Rivadeneira, Referente de la Red Nacional de Migrantes y Refugiados en Argentina, y el Frente Patria Migrante realizada en el marco de prácticas territoriales por el estudiante Abrahan Quisbert Alcon (26 de julio, 2019; material de cátedra).

Se parte de la idea de entender a la universidad como un actor estratégico y con la responsabilidad de aportar a la transformación y al desarrollo territorial en función de las demandas surgidas, respetando los saberes y la perspectiva de desarrollo endógeno.

Se entiende a la educación superior no sólo como un bien público destinado a producir conocimiento, sino también como un espacio que tiene la responsabilidad de ponerse en diálogo y de reconocer los saberes del territorio, a fin de generar estrategias de desarrollo y experiencias concretas de articulación con los y las estudiantes que la componen como agentes promotores de cambios, como jóvenes críticos con un rol decisivo en la sociedad que integran.

"Esta racionalidad metodológica involucra dimensiones que apuntan a comprender los fenómenos comunicacionales en la resistencia social y cómo éstos, son parte responsable del ordenamiento lógico de las estrategias utilizadas por los sujetos, en el proceso de extensión universitaria con perspectiva crítica." (Pascual, A; Thays, T.; Cornejo, M. 2020)

El territorio se entiende como el espacio de articulación con la Universidad en donde ésta no imparte conocimiento al Territorio sino que lo construye como diría Freire en la mediación de ambos con el mundo, con ese bagaje de saberes, conocimientos y contextos con el que cargan los actores. 

Universidad en donde ésta no imparte conocimiento al Territorio sino que lo construye como diría Freire en la mediación de ambos con el mundo, con ese bagaje de saberes, conocimientos y contextos con el que cargan los actores.

En este sentido, la práctica de extensión-articulación-educación que se propicia desde Prácticas Territoriales se comprende como el diálogo entre los y las actores que se construye de manera bidireccional, que surge de la mediación con el mundo que es todo lo que rodea y forma a un sujeto y cuyo rol esencial es: por un lado, conservar y por otro, transformar. Conservar aquellos conocimientos que circulan en la sociedad y darle las herramientas necesarias a los sujetos para que éstos mediante la reflexión puedan subjetivar esos contenidos y crear nuevos discursos dentro de una sociedad.

Esto es, un intercambio de saberes, que forman parte de la cultura, entre la comunidad y la Universidad a través de sus estudiantes y docentes.

Es así que se entiende a la cultura como el conjunto de resignificaciones propias que realiza un determinado grupo sobre los procesos sociales, en palabras de Néstor Canclini “la cultura abarca el conjunto de los procesos sociales de significación, o para decirlo de un modo más complejo, la cultura abarca el conjunto de los procesos sociales de producción, circulación y consumo de la significación en la vida social" (1997, pág. 35) por esta razón se cree esencial construir el objeto desde las voces y las problemáticas que fueron silenciadas durante mucho tiempo.

\section{LA CONSTRUCCIÓN COLECTIVA DE LA IDENTIDAD INSTITUCIONAL. LAS NUEVAS PREGUNTAS DE INVESTIGACIÓN.}

En el ejercicio de la integralidad y de la sistematización como enfoque nos posicionamos desde la experiencia vivida y registrada de los y las estudiantes en la construcción colectiva de la identidad institucional del IUNMa debido a que la pertenencia e identidad educativa del sujeto de la educación en Prácticas Territoriales, se construye en estrecha relación entre la academia-territorio.

En este sentido, es pertinente remarcar que la condición inaugural del territorio IUNMa fue punta de lanza para el trabajo de docencia y extensión, el cual se vio fuertemente marcado por las voces, identidades, historias y vivencias de adultos mayores y jóvenes estudiantes e integrantes de organizaciones sociales que co-construyen saberes y cargan de sentido la vida del Instituto Universitario Nacional "Madres de Plaza de Mayo". 

gural del territorio IUNMa fue punta de lanza para el trabajo de docencia y extensión, el cual se vio fuertemente marcado por las voces, identidades, historias y vivencias de adultos mayores y jóvenes estudiantes e integrantes de organizaciones sociales que co-construyen saberes y cargan de sentido la vida del Instituto Universitario Nacional "Madres de Plaza de Mayo".

En esta línea, se parte desde la idea de un sujeto/investigador subjetivo, es decir, de un sujeto construido socialmente e ideológicamente, un sujeto "puesto" desde las condiciones sociales, desde la organización del modo de producción, incluso dentro de la específica comunidad científica y el específico propio lugar en ella" (Follari, R. en Epistemología y Sociedad 2000).

La modalidad taller y el rol docente como facilitador de procesos educativos, la elaboraciòn de sus propios proyectos sociales, políticos, críticos y emancipatorios que se desarrollarán en los niveles de prácticas dos y tres permitieron el desarrollo de preguntas de investigación que dan cuenta de la necesidad de seguir profundizando y reflexionando para dar respuestas a dichos procesos cognitivos y de intervención social, que encuentra profundidad en el tercer año completo de la propuesta curricular de Prácticas Territoriales.

Así, surgen interrogantes que interpelan las prácticas curriculares y complejizan las estrategias formativas porque permiten delinear acciones de trabajo, donde el registro y análisis de las respuestas permitirán trazar nuevos horizontes de trabajo para el 2021/22: ¿Qué estrategias de enseñanza/aprendizaje vivenciadas como estudiantes y docentes permitieron conformar una identidad colectiva en la institución educativa IUNMa? ¿Cómo caracteriza el y la estudiante la institución educativa de las Madres? ¿Cómo caracterizan los y las estudiantes organizados en el Centro de Estudiantes su rol y función desde sus prácticas cotidianas en el IUNMa? ¿Qué valoración atribuyen los y las estudiantes a la enseñanza y aprendizaje de prácticas territoriales para el desarrollo de sus tareas?

En las ciencias sociales, a diferencia de las ciencias naturales, el objeto que se estudia es también un sujeto que, para nada, es neutral: tiene un contexto en el cual está inmerso, una historia, unas costumbres, una determinada identidad. Por ende, es clave entender el proceso de articulación con el territorio como un proceso de construcción dialógica, de intercambio de saberes en donde, como plantea Paulo Freire en Pedagogía del Oprimido, "no se hace de A para B o de A sobre $B$, sino de $A$ con B, con la mediación del mundo"

En esta línea, las organizaciones sociales del territorio ocupan un lugar activo en todo este proceso, y deberán ser co-protagonista en 
la ejecución y toma de decisiones en la vida universitaria. Por ello, forma parte de todo proceso, desde el momento de la identificación y construcción de la demanda y del problema, hasta la evaluación del impacto y su sustentabilidad en el tiempo.

Se considera que es indispensable remarcar el rol clave de los medios de comunicación como instituciones productoras y reproductoras de los discursos que circulan dentro de la sociedad.

"La comunicación tiene que darle visibilidad a los pobres, a los líderes sociales, como sujetos, pero tambièn a sus propuestas y a sus iniciativas. No habrá desarrollo verdadero sin nuevos actores visibles y reconocidos en el escenario social. La ciudadanía exige e impone de todos y todas por igual. Demanda también poner en tensión a lo diferente y educar acerca del valor de la diferencia. Desde una perspectiva comunicacional es necesario trabajar para garantizar la visibilidad de muchos que hoy "no existen" para la sociedad en su conjunto y porque son "invisibles" no son tomados en cuenta y desprovistos de todo poder real" (Uranga, Washington 2005).

\section{CONCLUSIONES PRELIMINARES}

En esta línea, con las y los estudiantes y la profesora de Prácticas Territoriales III, Mariana Castro, se analizó desde una mirada comunicacional y desde el enfoque de la extensión crítica al Instituto como territorio con actores y tramas complejas que lo habitan. Se propusieron diferentes estrategias para visibilizar trabajos de los y las estudiantes que recuperaban la voz de los y las "invisibles", los cuales fueron publicados en páginas web y redes sociales representativas de la actividad universitaria del IUNMa (MINERVA - Agencia de Noticias; @ estudiaenmadres)

Planificar y ejecutar la integralidad de las funciones sustantivas en la "Universidad de las Madres", nos genera, en principio, dos desafíos. Por un lado, el de generar las instancias de debate necesarias para confluir en un órgano que tome la forma de consejo social donde se promueva el diálogo, la cooperación y comunicación entre organizaciones de la sociedad y el gobierno de la universidad, con el objeto de promover estrategias de articulación y despliegue conjunto de actividades de investigación, docencia y extensión para la real transformación de las condiciones de vida del pueblo.

Por otro, es el generar diferentes mecanismos que permitan el crecimiento del Instituto en matrícula, retención y graduación de sus estudiantes, teniendo en cuenta que la carrera de comunicación aún no posee graduades. En esta línea, es que comprendemos a la extensión como una función clave para lograr este objetivo, "entendemos que sistematizar experiencias es un proceso que nos permite construir y recuperar desde la historia vivida en las experiencias, recuperar apren- 
dizajes y que sean significativos. Y no solamente para dar cuenta de lo que pasó en las experiencias, sino para orientar a dónde queremos impulsarlas (Isa, L., Zapata, N., \& Espinoza, J. 2019), pero esas experiencias a sistematizar provienen de un territorio mediado por el diálogo de saberes, de la disputa del mismo, de la tensión entre los y las actores.

Estas experiencias nos permiten discutir con la teoría, nos permite que la construcción de saberes sea participativo. Nos permite construir conocimiento con la sociedad y que los y las estudiantes sean protagonistas del acto educativo. En esta línea nos parece pertinente pensar que "la extensión (...) al ser introducida en el acto educativo normal, se convierte en un aliado para transformar el modelo pedagógico que en general ha sido profesionalizante (Errandonea, A: 1998) y no integral". (Arocena, R; Tommasino, H. y otros/as 2010).

Por lo tanto, podemos afirmar que cuando "hablamos de un nuevo acto educativo, hacemos referencia al diálogo permanente entre docencia, investigación y extensión, en donde cada uno se retroalimenta constantemente de la otra, enriqueciendo así la relación docente-estudiante, docente-formación contínua, estudiante-universidad-sociedad, universidad-entorno, universidad-sistema educativo, universidad-desarrollo", como sostuviera Jorge Orlando Castro quien fuera de esos docentes que no abundan, comprometido con las causas justas, que supo ser formador de formadores, que supo ser semilla regada por los territorios de toda nuestra América Latina, quien supo hacernos entender, comprender y vivenciar la importancia de la integración de las funciones de la universidad, para abrirla al pueblo desde cada una de las materias que integremos. 


\section{BIBLIOGRAFIA}

Canclini, Néstor García. (1997). "Cultura y comunicación: entre lo global y lo local”. Número 9 de Ediciones de periodismo y comunicación. Editor Facultad de Periodismo y Comunicación Social Universidad Nacional de la Plata. Procedencia del original Universidad de Texas. Digitalizado 18 Oct 2008 N. o de páginas 133 páginas.

Uranga, W. (2005). “Desarrollo, ciudadanía, democracia: aportes desde la comunicación". Buenos Aires: III Congreso Panamericano de Comunicación.

Follari, R. (2000). “Epistemología y sociedad. Acerca del debate contemporáneo". Santa Fe, Argentina, Homo Sapiens, Ediciones (Serie Estudios Sociales), 125 pp.

Erreguerena, F. (2020). "Las prácticas sociales educativas en la universidad pública: una propuesta de definición y esbozo de coordenadas teóricas y metodológicas". En Revista Masquedós N 5, Año 5. Secretaría de Extensión UNICEN. Tandil, Argentina.

Rinesi, E (2015). “Filosofía (y) política de la Universidad”. En ediciones UNGSIEC Colección Educación-Serie Universidad № 06144 páginas.

Pascual, A; Thays, T.; Cornejo, M. (2020). "Expresiones estudiantiles para la participación ciudadana en comunicación y salud: reflexiones sobre las prácticas socio educativas curriculares para la formación integral". En "Cuadernos de Extensión Universitaria de la UNLPam" (ISSN 2451-5930) - Convocatoria 2020. En prensa; 16 págs.

Remedí, E (1994). "Intervención. Mesa de trabajo I". Foros internacionales de análisis sobre Educación, EDUCAR, México.

Mata, M. C. (2005). “Condiciones objetivas y subjetivas para el desarrollo de la ciudadanía comunicativa". Córdoba (Argentina): Centro de Competencia en Comunicación para América Latina.

Teixeira, T. H. S. (2019). "Metodologia da Resistência. Tese.Programa de Pós-graduação em Comunicação em Estudos da Mídia". Universidade Federal do Rio Grande do Norte (UFRN). Natal.

Peralta M.I. [CPA UNLPam] (septiembre 2020). "La participación estudiantil en procesos de extensión: ¿por qué las y los estudiantes fueron y son motor de la extensión?" Ciclo web "La UNLPam y la Extensión, conceptos y experiencias desde el territorio" Recuperado en https://youtu.be/YHkjQOKoZGk

Castro, J. y Oyarbide, F. (Comps.) (2015). “Los caminos de la extensión en la universidad argentina". Santa Rosa: Universidad Nacional de La Pampa. 
Ley 26.995 de Creación del Instituto Universitario Nacional de Derechos Humanos "Madres de Plaza de Mayo". http://servicios.infoleg.gob.ar/infoleglnternet/anexos/235000-239999/238031/norma.htm

López, M. (2020). “Teoría y Metodología de la extensión universitaria: el caso argentine"- 1a ed. - San Salvador de Jujuy: El Siku. 203 pág. ISBN978-987-26670-2-3 Libro digital, PDF recuperado en https://www.researchgate.net/publication/341977630_TEORIA_Y_METODOLOGIA_DE_LA_EXTENSION_UNIVERSITARIA_EL_CASO_ARGENTINO

Rectorado Universidad de la República Montevideo URUGUAY (2010). "Hacia la reforma universitaria" \#10 LA EXTENSIÓN EN LA RENOVACIÓN DE LA ENSEÑANZA: ESPACIOS DE FORMACIÓN INTEGRAL.

Isa, L., Zapata, N., \& Espinoza, J. (2019). “Oscar Jara Holliday: «La extensión es el motor de la relación universidad-sociedad»". Extensión En Red, (10), e013. https://doi.org/10.24215/18529569e013

Arocena, Rodrigo; Tommasino, Humberto; Rodríguez, Nicolás; Sutz, Judith; Alvarez Pedrosian, Eduardo; Romano, Antonio. “Cuadernos de Extensión № 1: Integralidad: tensiones y perspectivas". 2010 recuperado en URI: http://beu.extension. unicen.edu.ar/xmlui/handle/123456789/168

Fernando S., Barrera, M. (2009). "El tizón encendido.Protesta social, conflicto y territorio en la Argentina de la posdictadura de Buenos Aires". Editorial El Colectivo.

Guillaza, V. (2018).“Intervención, territorios y actorxs sociales" [Material de clase]. En Taller de Prácticas territoriales, de la Licenciatura en Comunicación, IUNMa.

CV

*Lic. y Prof. en Comunicación Social, egresada de la UNLP; Esp. en divulgación científica título otorgado por la OEl; Docente Titular de Prácticas territoriales 1 del IUNMA; JTP regular en Producción y gestión de medios en la UNDAV. Investigadora en el GT CLACSO/ULEU Extensión Crítica: Teorías y Prácticas en América Latina y el Caribe.

**Prof en Comunicación Social, egresada de la UNLP. Maestranda en Comunicación y Educación por la UNLP. Docente Asociada de Prácticas Territoriales 2 del IUNMa. Adscripta graduada del Taller de Análisis de Comunicación en Instituciones Educativas de la FPyCS. Investigadora en el GT CLACSO/ULEU Extensión Crítica: Teorías y Prácticas en América Latina y el Caribe. 